Published in final edited form as:

Dig Dis Sci. 2018 September ; 63(9): 2267-2274. doi:10.1007/s10620-018-4964-0.

\title{
Hospital Cirrhosis Volume and Readmission in Patients with Cirrhosis in California
}

\author{
Mike Wei, Jason Ford, Qihan Li, Donghak Jeong, Allison J. Kwong, Mindie H. Nguyen, and \\ Matthew S. Chang
}

\author{
Keywords \\ Healthcare Cost and Utilization Project; Readmission; Cost Utilization; Cirrhosis; Hospital \\ Volume
}

\section{Introduction}

The economic burden of chronic liver disease and decompensated cirrhosis is significant, accounting for upwards of $\$ 2.5$ billion a year in healthcare-related spending in the United States alone.[1-4] As part of an ongoing effort to improve health care quality and consistency, it is essential to identify and understand gaps in the care of patients with cirrhosis. Readmission rates have been identified as a potential area of improvement.[2] Patients with decompensated cirrhosis patients experience hospital readmissions at higherthan-average rates, estimated in one study to be $14 \%$ at 7 days and up to $37 \%$ at 30 days.[59]

Reducing readmission rates not only improves quality of life and the burden of disease but has become essential in controlling healthcare-related expenditures in the context of valuebased compensation.[10-11] Several initiatives have been explored to reduce the rate of readmission, such as early outpatient follow-up, and the use of paper or electronic checklists for cirrhotic complications including upper gastrointestinal bleeding or hepatic encephalopathy. $[1,12,13]$

Several hospital factors been found to affect clinical outcomes for several medical conditions. Greater hospital volumes are associated with improved mortality for surgical procedures including heart valve replacement and nephrectomy as well as medical conditions such as congestive heart failure, pneumonia, and acute myocardial infarction. $[14,15]$ In congestive heart failure, increased hospital experience and expertise with cardiac management has been associated with reduced 30-day readmission rates.[16] Based on observations from this literature, our study leverages a large statewide database to evaluate the effect of hospital size and experience on readmission rates for patients with cirrhosis.

\section{Methods}

This study was conducted using data obtained through the Healthcare Cost and Utilization Project (HCUP), a database compiled and maintained by the Agency for Healthcare Research and Quality. Data were extracted from the State Inpatient Database (SID) for 
California between 2009 and 2011. Compared to the Nationwide Inpatient Sample, the national admission database, the California statewide database provides an added advantage of being able to follow patients across hospitalization records within the state via unique patient identifiers. Hospital characteristics (bed size, transplant center, urban/rural, teaching status) were obtained from the 2009 American Hospital Association Annual Survey Database (2009, Health Form, LLC).

Index hospitalizations for adults (age $\geq 18$ years) admitted with a diagnosis of cirrhosis were identified using the International Classification of Diseases, 9th Revision-Clinical Modification (ICD-9-CM) and validated protocols by Tapper et al. and Mellinger et al.[5,13] The specific ICD-9 codes used for cirrhosis were 571.2, 571.5, and 571.6. Exclusion criteria included mortality during the index hospitalization, an admission within 30 days prior to the index admission, records containing corrupted data, and history of liver transplant (ICD9CM V42.7, 50.5, 50.51, 50.59). (Figure 1).

The primary outcome measured was the 30-day readmission rate. To study the effect of hospital volume as a marker of experience for cirrhosis management, we defined hospital volume as the number of unique patients admitted for cirrhosis-related hospitalizations to each hospital. Cirrhosis-related hospitalizations were defined as any admission with associated ICD-9 code for cirrhosis. When arranging hospitals by increasing cirrhosis volume graphically, there was a marked increase in cirrhosis volume starting at the top $10 \%$ of hospitals (Figure 2), which served as a natural point to divide hospitals into higher (top $10 \%$ ) and lower (bottom 90\%) cirrhosis volume comparison groups. Additional patient variables analyzed included age, race/ethnicity, gender, homelessness, insurance status, length of stay, comorbidity score, clinical complications of cirrhosis as a marker for disease severity, hospital characteristics, and disposition. Comorbidity scores were represented by the Quan modification of Charlson-Deyo comorbidity score (Quan-Charlson-Deyo score [QCD]). The Charlson-Deyo comorbidity score is a well-established research scoring system based upon administrative ICD-9 data to characterize a patient's disease severity index. The Quan modification of Charlson-Deyo scoring has been demonstrated to provide better predictions of in-hospital mortality.[17]

Complications of cirrhosis were determined by ICD-9 codes for clinical hepatic decompensation, such as ascites (ICD9-CM 789.5x or ICD9-PR 54.91), hyponatremia (276.1), hepatic encephalopathy (572.2), esophageal varices (456.0, 456.1, 456.2), variceal hemorrhage (456.0, 456.2), end stage liver disease (572.8), jaundice (782.4), spontaneous bacterial peritonitis (SBP) [567.23], hepatorenal syndrome (572.4), hepatocellular carcinoma (155.0) and those that received transarterial chemoembolization (TACE) [ICD9PR 38.80, 38.86, 99.25) or transjugular intrahepatic portosystemic shunt (TIPS) [ICD9-PR 39.1]. Etiologies of cirrhosis including hepatitis B (702.x, 703.x, V02.61), hepatitis C (704.1, 705.x, 707.x, V02.62), fatty liver disease (571.8), alcoholic liver disease (571.0, 571.2-3, 303.x, 305.0) and primary sclerosing cholangitis (576.1) were also recorded. Other related variables included esophagogastroduodenoscopy (EGD) [ICD9-PR 45.13, 45.16, 45.19], dialysis (V45.11), ventilation as a proxy for ICU (96.70), paracentesis (ICD9-PR 54.91), systemic chemotherapy (99.25), as well as procedures to include destruction, ablation, or removal of liver tissue, including hepatectomy. Primary biliary cirrhosis was not 
included in the analysis as the ICD9 code (571.6) has not been well validated. We assessed for multicollinearity by testing for the variance inflation factor (VIF) and used a cut-off of 2.5 to indicate potential multicollinearity. Variables that had a VIF of 2.5 or greater were excluded from the final logistic regression model.

Using VisitLink, the unique patient identifier in the HCUP database, patients were tracked over time by their hospital admissions within California. Readmission was determined if there was admission within 30 days of discharge. Data management was performed using Statistical Analysis System ${ }^{\circledR}$ (SAS) 9.4 (Cary, NC), Python ${ }^{\circledR}$ (Wilmington, DE), and RStudio $^{\circledR}$ (Boston, MA).

\section{Statistical Analysis}

For all analyses, a p-value of $<0.05$ was considered significant, and all tests were 2-tailed. Multivariable logistic regression analysis was performed to assess the impact of hospital cirrhotic experience on readmission, with adjustment for potential confounders including age, race, gender, homelessness, insurance status, hospital size, hospital characteristics, comorbidity score, disposition, and complications as well as etiologies of cirrhosis. Statistical analyses were performed using SAS ${ }^{\circledR} 9.4$ (Cary, NC) and RStudio ${ }^{\circledR}$ (Boston, MA).

\section{Ethics Statement}

The study was determined to be exempt from review by the Stanford University Institutional Review Board. As such, the ethics committee at Stanford University approved the retrospective analysis of the patient data.

\section{Results}

There were a total of 170,784 eligible hospitalizations, representing 69,612 patients with cirrhosis at 405 hospitals, ranging from 1 to 1,687 patients with cirrhosis seen (Figure 1). The top $10 \%$ of hospitals by cirrhosis volume, serving a median of 747 patients with cirrhosis per hospital (interquartile range [IQR] 450) over the 3-year study period, included 41 hospitals and a total of 24,062 patients. The bottom $90 \%$ of hospitals, serving a median of 152.5 patients with cirrhosis per hospital (IQR 243.5), included 364 hospitals and a total of 45,550 patients. $52.7 \%$ of the readmissions were to the same hospital as the index hospitalization. The median number of hospital admissions per patient within the study period was 2 (IQR 2). The emergency department was the most frequent source of first admissions (79.4\%), followed by home and other (16.5\%), and other hospital transfer (2.5\%); separately, for subsequent admissions, the emergency department was the most frequent source (77.0\%), with home and other being less (13.3\%), and other hospital transfer being relatively more frequent $(7.5 \%)$ [Supplementary Table 1$]$.

There was similar mean age, gender and race between lower-volume (90\%) and highervolume (10\%) hospitals (Table 1). Compared to patients at higher volume hospitals, a larger proportion of patients at lower volume hospitals had Medicare (42.6 v. 36.0\%) and a smaller proportion had Medicaid (20.4 v. 25.6\%).

Dig Dis Sci. Author manuscript; available in PMC 2019 September 01. 
Between the two groups, patients from lower-volume hospitals demonstrated a slightly lower level of severity of patient comorbidities as calculated by the Quan-Charlson-Deyo score (average 6.54 v. 6.68). Patients with HCC were less likely to be admitted to a low-volume hospital (3.8 v. 8.7\%). Among etiologies of cirrhosis, there was slightly greater burden of alcoholic liver disease (53.1 v. $47.4 \%$ ) but lower burden of hepatitis C (34.8 v. $41.5 \%)$ for low-volume hospitals. Regarding disposition, patients from higher-volume hospitals had a lower proportion of patients discharged to home $(66.9 \mathrm{v} 74.3 \%)$ but higher proportion of discharge to other facilities, including skilled nursing facility and intermediate care facilities. The length of stay was not significantly different between lower-volume and higher-volume hospitals (6.10 v. 6.56 days). The 30-day readmission rate for lower-volume hospitals was $19.1 \%$, and $18.2 \%$ for higher-volume hospitals.

In the first multivariable logistic regression model that includes hospital cirrhosis patient volume without other hospital characteristics, admission to a hospital with lower cirrhosis patient volume was modestly associated with readmission within 30 days (odds ratio [OR] $1.05,95 \%$ confidence interval [CI] 1.01-1.10) after adjusting for sociodemographics, QCD score and complications related to cirrhosis (Supplementary Table 2). However, in our main multivariate logistic regression model that included additional hospital characteristics derived by linking data from the American Hospital Association survey database, we found that cirrhosis patient volume was no longer an independent risk factor for readmission (OR $0.97,95 \%$ CI 0.92-1.01), while liver transplant center status predicted against readmission within 30 days (OR $0.87,95 \%$ CI $0.80-0.94$ ) (Table 2).

Notably, Asian or Pacific Islanders (OR 1.19, 95\% CI 1.09-1.29) and Native Americans (OR $1.12,95 \%$ CI 1.06-1.18) were more likely to be readmitted compared to whites (Table 2). As expected, increasing Quan-Charlson-Deyo comorbidity score was also a predictor (OR $1.01,95 \% 1.00-1.02)$ of readmission, as were alcoholic liver disease, hepatitis $\mathrm{B}$ and hepatitis C. Patients with both hepatitis B and C did not experience increase in odds of readmission. Fatty liver disease decreased the odds of readmission (OR 0.79, 95\% CI 0.670.93). Primary sclerosing cholangitis did not demonstrate an increase in readmissions.

Among cirrhotic complications (Table 2), our model identified ascites (OR 1.61, 95\% CI: 1.53-1.69), hepatic encephalopathy (OR 1.47, 95\% CI 1.38-1.56), TIPS (OR 1.71, 95\% CI 1.38-2.10), hepatocellular carcinoma (OR 1.18, 95\% CI 1.07-1.29), hyponatremia, jaundice, paracentesis, and presence of ESLD as independent predictors of 30-day readmissions. The presence of variceal bleed, SBP, or hepatorenal syndrome did not increase odds of readmission. Patients that received liver surgery had lower odds of readmission (OR 0.31, 95\% CI: $0.20-0.45)$ but only 515 patients received surgery $(<0.01 \%)$.

In addition, urban hospitals increased odds of readmission (OR 1.22, 95\% CI 1.06-1.40) relative to rural hospitals (Table 2). Academic hospitals also experienced an increased odds of readmission (OR 1.14, 95\% CI 1.01-1.28). In terms of disposition, relative to discharging home, discharge to short term hospital (OR 6.57, 95\% CI 6.01-7.18) or other facility (such as skilled nursing, intermediate care) [OR 2.05, 95\% CI 1.94-2.17], against medical advice (OR 1.72, 95\% CI 1.53-1.94) increased odds of readmission, whereas discharge to home health care did not increase the odds of readmission (OR 1.05, 95\% CI 0.98-1.13). Hospital 
bed size had a VIF of 2.38-5.61 concerning for multicollinearity and as such was excluded from the final regression model.

\section{Discussion}

In summary, we leveraged the HCUP California State Inpatient Database to define readmission rates across California between 2009 and 2011 for patients with cirrhosis and identify risk factors for 30-day readmissions in this large, diverse cohort.[5] We have demonstrated that admission to a lower-volume hospital was not a risk factor for readmission with 30 days. Instead, sociodemographic factors and clinical complexity were instead stronger predictors of readmission. While hospital volume as a proxy for cirrhosis experience did not prove to be a statistically significant risk factor for readmission, liver transplant center status significantly decreased odds of readmission $(0.87,95 \%$ CI 0.80 0.94). Liver transplant centers comprised of 13 hospitals out of 405 hospitals (3.2\%), with median hospital volume 1,062 (IQR 1,246) patients. One possibility to explain the discrepancy is that being a liver transplant center may provide a degree of experience managing patients with cirrhosis that cannot be provided simply by volume alone.

The 30-day readmission rate was $18.8 \%$ for our study population. Prior estimates of 30-day readmission rates in patients with cirrhosis have ranged from 13 to $37 \% .[2,18,19]$ This wide range may be due to regional differences, or the fact that some of the studies were limited to a few academic centers. In our cohort, Native Americans were more likely to experience a readmission within 30-days compared to Caucasians (OR 1.12, 95\% CI 1.06-1.18), and with longer duration of admission. This is consistent with reports from the Centers for Disease Control and Prevention (CDC), in which liver disease is the fifth leading cause of mortality for Native Americans, compared to sixth for Hispanics, and outside the top 10 causes for Asian or Pacific Islanders, Caucasians, and African Americans.[20] Native Americans comprise $0.5 \%$ of the population in California, and yet accounted for $15.8 \%$ of the admissions in our study.[21]

This study has several limitations. Claims data are subject to error and selection bias. However, since the claims data also is the vehicle by which hospitals are reimbursed, there is particular motivation for diagnoses to be accurately reflected. The database also lacks lab results and other clinical information, but this to some measure is compensated for by the Quan modification of the Charlson-Deyo score, a research tool that serves as a reliable surrogate for co-morbidities. While Model for End-Stage Liver Disease (MELD) score is not available through this data set to account for the severity of liver disease, we have accounted for complications of cirrhosis using ICD-9 codes. Additionally, HCUP California does not provide more detail beyond discharge home, short-term hospital transfer, other facility transfers including skilled nursing and intermediate care facility, and home health care. The California dataset does not provide data to account for patients that were discharged to home hospice and death after discharge, which is a competing risk for readmission. However, the home health care cohort, which encompassed home hospice, accounted for only $10.2 \%$ of patients, and was not statistically significant (OR 1.05, 95\% CI 0.98-1.13). Finally, primary biliary cirrhosis was not included in the analysis as the only available code for this diagnosis (571.6) was not specific enough; however, the impact was likely limited given the relatively 
small numbers of patients for the available code $(n=1,335)$. The findings of this study may serve as a barometer for hospitals as they innovate strategies to keep patients with cirrhosis healthy and out of hospitals, improving quality and driving down cost of care. Many factors relevant to readmission were not modifiable, but identification of specific actionable risk factors may help hospitals target resources appropriately. We found that hospital cirrhosis patient volume was not a risk factor for readmission in California. We instead found that medically complicated patients with cirrhosis at hospitals that were not liver transplant centers are at increased risk for readmission and may benefit from additional support or even transfer to a transplant center to prevent readmission.

\section{Supplementary Material}

Refer to Web version on PubMed Central for supplementary material.

\section{References}

1. Kanwal F, Kramer J, Asch SM, et al. An explicit quality indicator set for measurement of quality of care in patients with cirrhosis. Clin Gastroenterol Hepatol. 2010; 8:709-717. [PubMed: 20385251]

2. Volk ML, Tocco RS, Bazick J, et al. Hospital readmissions among patients with decompensated cirrhosis. Am J Gastroenterol. 2012; 107:247-252. [PubMed: 21931378]

3. Kim WR, Brown RS, Terrault NA, et al. Burden of liver disease in the United States: summary of a workshop. Hepatology. 2002; 36:227-242. [PubMed: 12085369]

4. Neff GW, Duncan CW, Schiff ER. The current economic burden of cirrhosis. Gastroenterol Hepatol. 2011; 7:661-671.

5. Tapper EB, Halbert B, Mellinger J. Rates of and Reasons for Hospital Readmissions in Patients With Cirrhosis: A Multistate Population-based Cohort Study. Clin Gastroenterol Hepatol. 2016; 14:1181-1188.e1182. [PubMed: 27085758]

6. National Center for Health Statistics. Hospital Utilization (in non-Federal short-stay hospitals). CDC/National Center for Health Statistics; 2016. Available at: https:/www.cdc.gov/nchs/fastats/ hospital.htm. Accessed July 5, 2017

7. Jencks SF, Williams MV, Coleman EA. Rehospitalizations among patients in the Medicare fee-forservice program. N Engl J Med. 2009; 360:1418-1428. [PubMed: 19339721]

8. Miller ME. Report to the Congress: Reforming the Delivery System. Medicare Payment Advisory Commission; Washington, DC: 2008.

9. Jencks SF. Defragmenting care. Ann Intern Med. 2010; 153:757-758. [PubMed: 21135299]

10. Hansen LO, Young RS, Hinami K, et al. Interventions to reduce 30-day rehospitalization: a systematic review. Ann Intern Med. 2011; 155:520-528. [PubMed: 22007045]

11. Tapper EB, Volk M. Strategies to Reduce 30-Day Readmissions in Patients with Cirrhosis. Curr Gastroenterol Rep. 2017; 19:1. [PubMed: 28101791]

12. Kanwal F, Asch SM, Kramer JR, et al. Early outpatient follow-up and 30-day outcomes in patients hospitalized with cirrhosis. Hepatology. 2016; 64:569-581. [PubMed: 26991920]

13. Mellinger JL, Richardson CR, Mathur AK, et al. Variation among United States hospitals in inpatient mortality for cirrhosis. Clin Gastroenterol Hepatol. 2015; 13:577-584. [PubMed: 25264271]

14. Birkmeyer JD, Siewers AE, Finlayson EV, et al. Hospital volume and surgical mortality in the United States. N Engl J Med. 2002; 346:1128-1137. [PubMed: 11948273]

15. Ross JS, Normand SL, Wang Y, et al. Hospital volume and 30-day mortality for three common medical conditions. N Engl J Med. 2010; 362:1110-1118. [PubMed: 20335587]

16. Joynt KE, Orav EJ, Jha AK. The association between hospital volume and processes, outcomes, and costs of care for congestive heart failure. Ann Intern Med. 2011; 154:94-102. [PubMed: 21242366] 
17. Quan H, Sundararajan V, Halfon P, et al. Coding algorithms for defining comorbidities in ICD-9CM and ICD-10 administrative data. Med Care. 2005; 43:1130-1139. [PubMed: 16224307]

18. Berman K, Tandra S, Forssell K, et al. Incidence and predictors of 30-day readmission among patients hospitalized for advanced liver disease. Clin Gastroenterol Hepatol. 2011; 9:254-259. [PubMed: 21092762]

19. Bajaj JS, Reddy KR, Tandon P, et al. The 3-month readmission rate remains unacceptably high in a large North American cohort of patients with cirrhosis. Hepatology. 2016; 64:200-208. [PubMed: 26690389]

20. Heron M. Deaths: Leading Causes for 2013. Natl Vital Stat Rep. 2016; 65:1-95.

21. U.S. Census Bureau. QuickFacts. California: U.S Census Bureau, U.S. Department of Commerce; 2017. Available at: https://www.census.gov/quickfacts/. Accessed July 5, 2017 


\section{Excluded:}

Corrupt Data: 112,476 visits

Age<18: 2,307,151 visits

Diff Dx: 9,273,797 visits

\section{Patients with Cirrhosis}

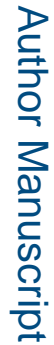

\section{Excluded:}

Admitted before $2009(1,135)$

Visits in January 2009 (1,527)

Visit in Jan 2009 with subsequent visit greater than 30 days $(1,101)$

First admission in December $2011(3,842)$

Death during index hospitalization $(5,768)$

Corrupt length of stay (8)

Negative days between $1^{\text {st }}$ and $2^{\text {nd }}$ visits (38)

Prior liver transplant (436) with cirrhosis

Figure 1.

Process of selection and exclusion of patients for the study 


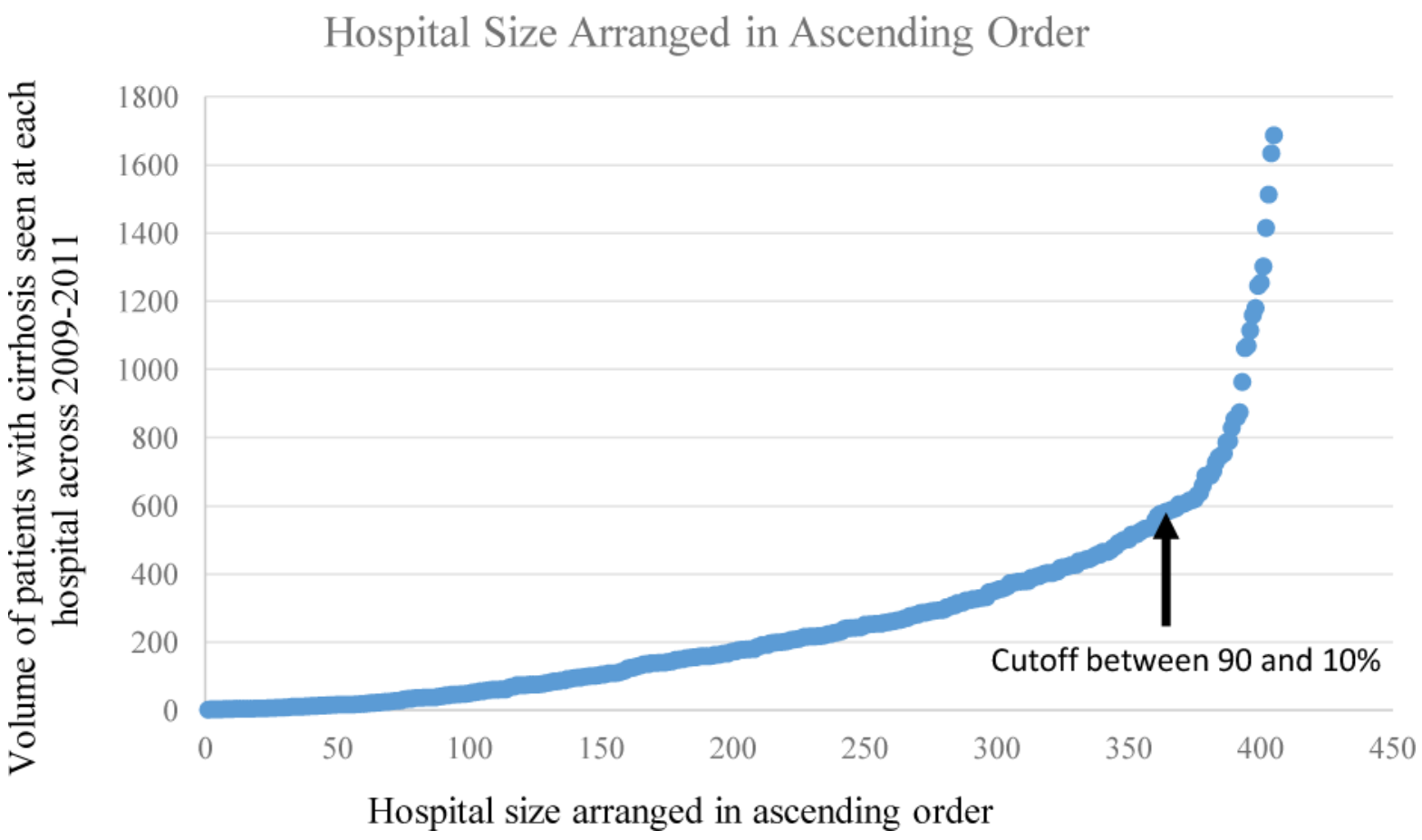

Figure 2.

Volume of patients with cirrhosis seen at each hospital between 2009-2011 arranged in ascending order 
Table 1

Cohort Characteristics by Hospital Cirrhosis Volume 2009-2011

\begin{tabular}{|c|c|c|}
\hline$N=69,612$ Patients & $\begin{array}{l}\text { Bottom 90\% Hospital Cirrhosis } \\
\text { Volume } \\
\text { N=45550 }\end{array}$ & $\begin{array}{l}\text { Top 10\% Hospital Cirrhosis } \\
\text { Volume } \\
\text { N=24062 }\end{array}$ \\
\hline Readmission within 30 days (\%) & $8,700(19.1 \%)$ & $4,390(18.2 \%)$ \\
\hline $\begin{array}{l}\text { Median number of unique patients with cirrhosis per hospital } \\
\text { (interquartile range) }\end{array}$ & $152.5(43-286.5)$ & $747(619-1,069)$ \\
\hline \multicolumn{3}{|l|}{ Patient Sociodemographic } \\
\hline Mean Age, years ( \pm SD) & $60.12( \pm 12.9)$ & $58.17( \pm 12.4)$ \\
\hline Female $(\%)$ & $17,730(38.9)$ & $8,781(36.5)$ \\
\hline \multicolumn{3}{|l|}{ Race/Ethnicity } \\
\hline Caucasian $(\%)$ & $31,812(69.8)$ & $16,489(68.5)$ \\
\hline African American (\%) & $3,180(7.0)$ & $1,644(6.8)$ \\
\hline Hispanic (\%) & $349(0.8)$ & $152(0.6)$ \\
\hline Asian or Pacific Islander (\%) & $2,918(6.4)$ & $1,539(6.4)$ \\
\hline Native American (\%) & $6,990(15.3)$ & $4,042(16.8)$ \\
\hline Other $(\%)$ & $300(0.7)$ & $195(0.8)$ \\
\hline \multicolumn{3}{|l|}{ Payment Type } \\
\hline Medicare $(\%)$ & $19,404(42.6)$ & $8,672(36.0)$ \\
\hline Medicaid (\%) & $9,308(20.4)$ & $6,152(25.6)$ \\
\hline Private Insurance (\%) & $10,426(22.9)$ & $4,750(19.7)$ \\
\hline Self-Pay (\%) & $3,330(7.3)$ & $1,763(7.3)$ \\
\hline Other $(\%)$ & $3,069(6.7)$ & $2,724(11.3)$ \\
\hline Homelessness (\%) & $453(1.0)$ & $401(1.7)$ \\
\hline \multicolumn{3}{|l|}{ Clinical Characteristics } \\
\hline Average Quan-Charlson-Deyo score $( \pm \mathrm{SD})$ & $6.54( \pm 4.2)$ & $6.68( \pm 4.3)$ \\
\hline Average length of stay $( \pm \mathrm{SD})$ & $6.10( \pm 8.13)$ & $6.56( \pm 9.47)$ \\
\hline \multicolumn{3}{|l|}{ Complications of liver disease } \\
\hline Ascites $(\%)$ & $13,210(29.0)$ & $7,115(29.6)$ \\
\hline Hepatic encephalopathy (\%) & $5,273(11.6)$ & $2,223(9.2)$ \\
\hline Esophageal varices (\%) & $1,854(4.1)$ & $957(4.0)$ \\
\hline Variceal hemorrhage $(\%)$ & $3,593(7.9)$ & $1,679(7.0)$ \\
\hline Hepatorenal syndrome (\%) & $692(1.5)$ & $502(2.1)$ \\
\hline Hepatocellular carcinoma (\%) & $1,710(3.8)$ & $2,086(8.7)$ \\
\hline Jaundice $(\%)$ & $1,128(2.5)$ & $437(1.8)$ \\
\hline TIPS $(\%)$ & $211(0.5)$ & $258(1.1)$ \\
\hline Paracentesis (\%) & $7,936(17.4)$ & $4,869(20.2)$ \\
\hline $\operatorname{SBP}(\%)$ & $855(1.9)$ & $575(2.4)$ \\
\hline Hyponatremia (\%) & $6,697(14.7)$ & $3,360(14.0)$ \\
\hline $\operatorname{ESLD}(\%)$ & $2,105(4.6)$ & $1,122(4.7)$ \\
\hline EGD $(\%)$ & $7,576(16.6)$ & 3,837 (15.9) \\
\hline
\end{tabular}

Dig Dis Sci. Author manuscript; available in PMC 2019 September 01. 


\begin{tabular}{|c|c|c|c|}
\hline \multirow{8}{*}{ 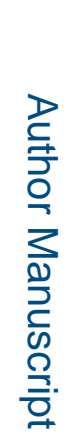 } & $\mathrm{N}=69,612$ Patients & $\begin{array}{l}\text { Bottom 90\% Hospital Cirrhosis } \\
\text { Volume } \\
\text { N=45550 }\end{array}$ & $\begin{array}{l}\text { Top 10\% Hospital Cirrhosis } \\
\text { Volume } \\
\text { N=24062 }\end{array}$ \\
\hline & TACE $(\%)$ & $239(0.5)$ & $550(2.3)$ \\
\hline & Systemic chemotherapy (\%) & $235(0.5)$ & $549(2.3)$ \\
\hline & Liver surgery $(\%)$ & $148(<0.01)$ & $367(<0.01)$ \\
\hline & Etiology of liver disease & & \\
\hline & Alcoholic liver disease (\%) & $23,388(53.1)$ & $11,414(47.4)$ \\
\hline & Hepatitis B (\%) & $5,133(11.3)$ & $3,016(12.5)$ \\
\hline & Hepatitis C (\%) & $15,853(34.8)$ & 9,985 (41.5) \\
\hline \multirow{12}{*}{ 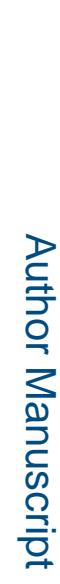 } & Hepatitis B and C (\%) & $1,829(4.0)$ & $1,195(5.0)$ \\
\hline & Fatty liver disease (\%) & $841(1.8)$ & $469(1.9)$ \\
\hline & $\operatorname{PSC}(\%)$ & $311(0.7)$ & $264(1.1)$ \\
\hline & Severity of illness & & \\
\hline & Ventilation & $6(<0.01)$ & $3(<0.01)$ \\
\hline & Dialysis & $1,100(2.4)$ & $533(2.2)$ \\
\hline & Hospital characteristics & & \\
\hline & Academic hospital & $790(1.7)$ & $1107(4.6)$ \\
\hline & Rural & $1,383(3.0)$ & $0(0.0)$ \\
\hline & Urban & $43,389(96.4)$ & $24,062(100.0)$ \\
\hline & Liver transplant & $275(0.6)$ & $5,387(22.4)$ \\
\hline & $<100$ hospital beds & $4,700(10.3)$ & $0(0.0)$ \\
\hline \multirow{12}{*}{ 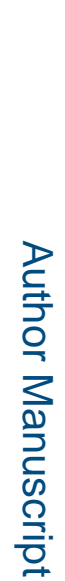 } & 100-199 beds & $15,709(34.5)$ & $276(0.1)$ \\
\hline & $200-299$ beds & $13,144(28.9)$ & $1,781(7.4)$ \\
\hline & $300-399$ beds & $9,588(21.0)$ & $6,448(26.8)$ \\
\hline & $400-499$ beds & $1,996(4.4)$ & $7,103(29.5)$ \\
\hline & $>500$ hospital beds & $135(0.3)$ & $8,454(35.1)$ \\
\hline & Disposition & & \\
\hline & Home ${ }^{*}$ & $30,463(66.9)$ & $17,871(74.3)$ \\
\hline & Transfer to short-term hospital & $1,774(3.9)$ & $540(2.2)$ \\
\hline & Transfer to other facility & $7,040(15.5)$ & $2,723(11.3)$ \\
\hline & Home health care & $4,843(10.6)$ & $2,256(9.4)$ \\
\hline & Against medical advice & $1,176(2.6)$ & $549(2.3)$ \\
\hline & Other & $253(0.6)$ & $121(0.5)$ \\
\hline
\end{tabular}

$\mathrm{SD}=$ Standard deviation

*Home: this HCUP-created category is formally routine disposition, which includes disposition to home or to court/law enforcement.

Dig Dis Sci. Author manuscript; available in PMC 2019 September 01. 
Table 2

Multivariate Analysis of Factors Affecting Readmission Within 30 Days

\begin{tabular}{|c|c|c|c|}
\hline & Odds Ratio & $95 \% \mathrm{CI}$ & P-value \\
\hline \multicolumn{4}{|l|}{ Hospital Volume } \\
\hline Top $10 \%$ & 1.00 & & \\
\hline Bottom 90\% & 0.97 & $0.92-1.01$ & 0.15 \\
\hline Age (per 10 years) & 1.00 & $1.00-1.00$ & 0.07 \\
\hline Female & 1.02 & $0.98-1.07$ & 0.29 \\
\hline \multicolumn{4}{|l|}{ Race/Ethnicity } \\
\hline Caucasian & 1.00 & & \\
\hline African American & 1.00 & $0.92-1.08$ & 0.96 \\
\hline Hispanic & 1.14 & $0.90-1.43$ & 0.26 \\
\hline Asian or Pacific Islander & 1.19 & $1.09-1.29$ & $<0.01$ \\
\hline Native American & 1.12 & $1.06-1.18$ & $<0.01$ \\
\hline \multicolumn{4}{|l|}{ Payment Type } \\
\hline Private & 1.00 & & \\
\hline Medicare & 1.13 & $1.07-1.19$ & $<0.01$ \\
\hline Medicaid & 1.17 & $1.11-1.24$ & $<0.01$ \\
\hline Self-Pay & 0.89 & $0.81-0.97$ & $<0.01$ \\
\hline Homelessness & 1.21 & $1.01-1.44$ & $\mathbf{0 . 0 3}$ \\
\hline \multicolumn{4}{|l|}{ Clinical Characteristics } \\
\hline Quan-Charlson-Deyo score & 1.01 & $1.00-1.02$ & $<0.01$ \\
\hline Length of stay & 1.00 & $0.99-1.00$ & $<0.01$ \\
\hline \multicolumn{4}{|l|}{ Complications of liver disease } \\
\hline Ascites & 1.61 & $1.53-1.69$ & $<0.01$ \\
\hline Hepatic encephalopathy & 1.47 & $1.38-1.56$ & $<0.01$ \\
\hline Esophageal varices & 1.09 & $0.98-1.20$ & 0.10 \\
\hline Variceal hemorrhage & 1.06 & $0.98-1.14$ & 0.16 \\
\hline Hepatorenal syndrome & 1.00 & $0.87-1.14$ & 0.95 \\
\hline Hepatocellular carcinoma & 1.18 & $1.07-1.29$ & $<0.01$ \\
\hline Jaundice & 1.29 & $1.14-1.45$ & $<0.01$ \\
\hline TIPS & 1.71 & $1.38-2.10$ & $<0.01$ \\
\hline Paracentesis & 1.22 & $1.17-1.28$ & $<0.01$ \\
\hline SBP & 1.12 & $0.99-1.27$ & 0.06 \\
\hline Hyponatremia & 1.27 & $1.21-1.34$ & $<0.01$ \\
\hline ESLD & 1.20 & $1.10-1.32$ & $<0.01$ \\
\hline EGD & 1.07 & $1.02-1.13$ & 0.01 \\
\hline TACE & 1.16 & $0.39-2.87$ & 0.76 \\
\hline Systemic chemotherapy & 0.71 & $0.28-2.16$ & 0.49 \\
\hline Liver surgery & 0.31 & $0.20-0.45$ & $<0.01$ \\
\hline
\end{tabular}

Dig Dis Sci. Author manuscript; available in PMC 2019 September 01. 


\begin{tabular}{|llll|}
\hline & Odds Ratio & $\mathbf{9 5 \%}$ CI & P-value \\
\hline Alcoholic liver disease & 1.14 & $\mathbf{1 . 0 9}-\mathbf{1 . 1 9}$ & $<\mathbf{0 . 0 1}$ \\
Fatty liver disease & 0.79 & $\mathbf{0 . 6 7 - 0 . 9 3}$ & $<\mathbf{0 . 0 1}$ \\
Hepatitis B & 1.03 & $0.95-1.11$ & 0.49 \\
Hepatitis C & 1.14 & $\mathbf{1 . 0 8 - 1 . 1 9}$ & $<\mathbf{0 . 0 1}$ \\
Hepatitis B and C & 1.09 & $0.96-1.23$ & 0.19 \\
PSC & 0.98 & $0.78-1.23$ & 0.89 \\
Severity of illness & & & \\
Ventilation & 2.00 & $0.45-8.18$ & 0.34 \\
Dialysis & 1.30 & $\mathbf{1 . 1 5}-\mathbf{1 . 4 7}$ & $<\mathbf{0 . 0 1}$ \\
Hospital characteristics & & & \\
Academic hospital & 1.14 & $\mathbf{1 . 0 1 - 1 . 2 8}$ & $\mathbf{0 . 0 4}$ \\
Rural & 1.00 & & \\
Urban & 1.22 & $\mathbf{1 . 0 6 - 1 . 4 0}$ & $<\mathbf{0 . 0 1}$ \\
Liver transplant & 0.87 & $\mathbf{0 . 8 0 - 0 . 9 4}$ & $<\mathbf{0 . 0 1}$ \\
Disposition & & & \\
Home & 1.00 & & \\
Transfer to short-term hospital & 6.57 & $\mathbf{6 . 0 1 - 7 . 1 8}$ & $<\mathbf{0 . 0 1}$ \\
Transfer to other facility & 2.05 & $\mathbf{1 . 9 4 - 2 . 1 7}$ & $<\mathbf{0 . 0 1}$ \\
Home health care & 1.05 & $\mathbf{0 . 9 8 - 1 . 1 3}$ & $\mathbf{0 . 1 5}$ \\
Against medical advice & 1.72 & $\mathbf{1 . 5 3 - 1 . 9 3}$ & $<\mathbf{0 . 0 1}$ \\
\hline
\end{tabular}

*Home: this HCUP-created category is formally routine disposition, which includes disposition to home or to court/law enforcement. 